\title{
Selectivity as a Function of Membrane Thickness: Gas Separation and Pervaporation
}

\author{
G. H. KOOPS, ${ }^{1}$ J. A. M. NOLTEN, ${ }^{1}$ M. H. V. MULDER, ${ }^{2, *}$ and C. A. SMOLDERS ${ }^{1}$ \\ 'Industrial Products Research Institute, 1-1-4, Higashi, Tsukuba, Ibaraki 305, Japan; ${ }^{2}$ University of Twente, P.O. Box \\ 217, 7500 AE Enschede, The Netherlands
}

\begin{abstract}
SYNOPSIS
In this article, the pervaporation selectivity as a function of the membrane thickness is studied for the dehydration of acetic acid. From this study, it appeared that the selectivity of polysulfone (PSF), poly (vinyl chloride) (PVC), and polyacrylonitrile (PAN) decreases with decreasing membrane thickness, below a limiting value of about $15 \mu \mathrm{m}$. However, in the case of gas separation, the selectivity of PSF membranes is independent of the membrane thickness. This phenomenon could not be explained by a difference in membrane morphology, sorption resistance, thermodynamic interaction, or coupling. It is believed that the decrease in selectivity for thin membranes has to be attributed to defects induced during pervaporation. These defects, crazes (and cracks), result from a reduced value of the critical strain, due to sorption of acetic acid/water and stresses between the polymer chains, due to a concentration gradient across the membrane. (C) 1994 John Wiley \& Sons, Inc.
\end{abstract}

\section{INTRODUCTION}

Transport of gases, vapors, and liquids through homogeneous films can be described by a "solutiondiffusion mechanism" as developed by Lonsdale et al. ${ }^{1}$ According to this model, transport takes place by sorption of the components into the membrane material at the feed side, followed by diffusion through the membrane, due to a chemical potential difference across the membrane and desorption at the permeate side. Fick's law has been used as a basis for this model, which describes the component flux through the membrane as being proportional to the reciprocal membrane thickness. Because this relation is valid for both components of a binary mixture, selectivity should be independent of the membrane thickness. This should be the case for liquid and gas mixtures.

In the case of pervaporation, the influence of membrane thickness on selectivity and flux was only studied by a few people. Binning et al. ${ }^{2}$ were one of the first and they concluded that the flux of a mixture of $n$-heptane and isooctane $(50 / 50 \mathrm{vol} \%)$

* To whom correspondence should be addressed. Journal of Applied Polymer Science, Vol. 53, 1639-1651 (1994) (C) 1994 John Wiley \& Sons, Inc. through a plastic film was proportional to the reciprocal membrane thickness and the selectivity was indeed independent of the thickness for membrane thicknesses in the range of $20-50 \mu \mathrm{m}$.

Brun et al. ${ }^{3}$ studied the influence of membrane thickness on selectivity using nitrile rubber membranes and a 60/40 mixture of butadiene and isobutene. They concluded that the selectivity was constant above a membrane thickness of $100 \mu \mathrm{m}$; a lower selectivity for membrane thicknesses of around $17 \mu \mathrm{m}$ was observed, especially at relatively high downstream pressures. This phenomenon was explained by assuming that elastomers are composed of very thin grains $(0.5-5 \mu \mathrm{m})$, which results in the formation of micropores in the membrane. Diffusion through these micropores becomes important in the case of thin membranes, resulting in a reduction of selectivity; the effect of these pores can be neglected in the case of thick membranes. Compaction of the membrane material due to a high pressure applied on the film results in the disappearance of micropores. Nevertheless, an influence of membrane thickness on selectivity could still be observed.

Spitzen et al. ${ }^{4}$ studied the separation of water/ ethanol mixtures as a function of the membrane thickness using polyacrylonitrile (PAN) as membrane material. They observed that at thicknesses 
below $20 \mu \mathrm{m}$ the selectivity dropped drastically. Reduction of the evaporation rate of the solvent from the casted film resulted in somewhat higher selectivities for thin membranes, but still a drop in selectivity could be observed with decreasing membrane thickness. Spitzen et al. attributed this mainly to artifacts in the membranes.

In another publication, ${ }^{5}$ the preparation of asymmetric integrally skinned polysulfone hollowfiber membranes was described. The selectivity of these membranes, with top-layer thicknesses of 0.8 $1.5 \mu \mathrm{m}$, for the dehydration of a $80 / 20 \mathrm{wt} \%$ acetic acid/water solution at $80^{\circ} \mathrm{C}$ varied between $\alpha=60$ and 80 , whereas a selectivity of $\alpha=620$ has been measured as the intrinsic selectivity of polysulfone.

Aptel et al. ${ }^{6}$ observed a reduction of selectivity as a function of decreasing membrane thickness for grafted polytetrafluoroethylene films using a water/ dioxane mixture and Mulder ${ }^{7}$ observed the same behavior for cellulose acetate membranes using a $50 / 50$ wt $\%$ mixture of ethanol and water.

It can be concluded that the transport of liquid mixtures does not (always) obey Fick's law since the selectivity is a function of the membrane thickness. It seems that a minimum thickness is required to obtain intrinsic selectivity, and below this thickness, selectivity starts to decrease with decreasing thickness.

On the other hand, for gas separation, some investigators showed ${ }^{8,9}$ that the intrinsic selectivity can be maintained even for thicknesses less than $500 \mathrm{~nm}$. This was found for quite a number of different materials such as polysulfone, polyimide, polyetherimide, modified poly (phenylene oxide), and polycarbonate. Although transport in both pervaporation and gas separation takes place according to the solution-diffusion model, there seem to be inherent differences, due to differences in affinity of gases and liquids toward polymers.

In this article, the pervaporation selectivity is studied as a function of the membrane thickness for three different materials, viz., polysulfone (PSF), poly (vinyl chloride) (PVC), and polyacrylonitrile (PAN), and the decline in selectivity below a certain minimum thickness will be discussed. Except for pervaporation, PSF and PVC have also been tested for gas separation as a function of membrane thickness and a comparison between gas separation and pervaporation has been made with respect to selectivity. The chemical stability of the polymers used was satisfactory, as could be deduced from determination of the weight decrease and of the change in molecular weight after exposure over a long period of time (2-7 days) in 90 and $99 \%$ of acetic acid at $80^{\circ} \mathrm{C} .{ }^{10}$

\section{EXPERIMENTAL}

\begin{abstract}
Materials
Polyacrylonitrile (PAN-7A) was obtained from DuPont, polysulfone (PSF, Udel P3500) from Amoco, and poly (vinyl chloride) (PVC, high MW) from Aldrich. The solvents $N, N$-dimethylformamide (for PAN and PSF) and tetrahydrofuran (for PVC) were of analytical grade and obtained from Merck.
\end{abstract}

\section{Membrane Preparation}

Membranes were prepared by solution casting on a glass plate followed by evaporation of the solvent in a nitrogen atmosphere. After the solvent had evaporated, the membranes were further dried in a vacuum oven at $80^{\circ} \mathrm{C}$ ( PVC and PAN) and at $150^{\circ} \mathrm{C}$ (PSF) for 1 week, before they were measured for pervaporation. Only the PSF membranes were applied to a heat treatment above the glass transition temperature $\left(1 \mathrm{~h}\right.$ at $\left.210^{\circ} \mathrm{C}\right)$ to improve their stability against environmental or solvent-induced stresscracking.

\section{Gas Separation}

Gas separation experiments were carried out using a $75 / 25$ vol $\% \mathrm{CH}_{4} / \mathrm{CO}_{2}$ mixture at room temperature and a pressure difference across the membranes of 6 bars. The setup used is totally automized and the permeability was calculated by an accurate measurement of the pressure increase with time in a calibrated volume at the downstream side of the membrane. The selectivity was determined by gas chromatographic analysis of the permeate samples.

\section{Pervaporation}

Pervaporation experiments were carried out using the same setup as described by Mulder et al. ${ }^{11}$ The permeate side was maintained at a pressure of 0.1 $0.5 \mathrm{mmHg}$ by a Crompton Parkinson vacuum pump. The pressure was measured by an Edwards pirani meter. The permeate pressure was kept constant during all the measurements. Permeate samples were taken every hour, during $8 \mathrm{~h}$. Steady state was normally obtained after $2-3 \mathrm{~h}$. The permeate was analyzed using a Varian 3700 gas chromatograph 
filled with a Poropack Q column at a temperature of $190^{\circ} \mathrm{C}$.

\section{RESULTS AND DISCUSSION}

\section{Gas Separation}

Asymmetric integrally skinned PSF hollow-fiber membranes as well as homogeneous PSF and PVC membranes with variable thicknesses were measured for the separation of a $\mathrm{CH}_{4} / \mathrm{CO}_{2}$ gas mixture. The selectivity and $P / l$ values of four different PSF hollow-fiber membranes are given in Table $I$.

The hollow-fiber membranes represented in $\mathrm{Ta}$ ble I differ from each other with respect to the polymer concentration and additive concentration used in the spinning dope. The effective membrane thickness of the hollow fibers has been calculated from the $P / l$ values and a permeability of 8.3 Barrers for PSF, which was obtained from measurements using homogeneous membranes with known thicknesses (Table II). In Table II, the selectivity and permeability of homogeneous PSF and PVC membranes are represented as a function of the membrane thickness.

From the results in Tables I and II, it can be concluded that for PSF the selectivity for the separation of $\mathrm{CO}_{2} / \mathrm{CH}_{4}$ gas mixtures is independent of the membrane thickness. In the case of PVC, the intrinsic selectivity could not be obtained anymore below a certain thickness ( $\pm 4 \mu \mathrm{m}$ ).

This can be explained by the relatively large defects in the PVC film introduced during evaporation of the solvent, which can be seen from the atomic force microscope pictures represented in Figure 1. Defects with a depth of more than $0.5 \mu \mathrm{m}$ could be observed. Due to the low permeability of PVC, a small single defect already has a large effect on the

Table I Selectivity and $P / l$ Values of Different PSF Hollow-Fiber Membranes as a Function of the Effective Thickness

\begin{tabular}{cccc}
\hline $\begin{array}{c}\text { Membrane } \\
\text { No. }\end{array}$ & Selectivity & $P / l^{\mathbf{a}}\left(\mathrm{CO}_{2}\right)$ & $\begin{array}{c}\text { Effective } \\
\text { Thickness } \\
(\mu \mathrm{m})\end{array}$ \\
\hline 1 & 30.3 & 3.7 & 2.2 \\
2 & 33.0 & 4.9 & 1.7 \\
3 & 30.8 & 6.5 & 1.3 \\
4 & 33.1 & 10.3 & 0.8 \\
\hline
\end{tabular}

${ }^{a} P / l: 10^{-6} \mathrm{~cm}^{3} / \mathrm{cm}^{2} \mathrm{~s} \mathrm{cmHg}$.
Table II Selectivity and $P / l$ Values of Homogeneous PSF and PVC Membranes as a Function of the Membrane Thickness

\begin{tabular}{cccc}
\hline Membrane & $\begin{array}{c}\text { Thickness } \\
(\mu \mathrm{m})\end{array}$ & $\begin{array}{c}\text { Selectivity }^{\mathrm{a}} \\
\alpha\left(\mathrm{CO}_{2} / \mathrm{CH}_{4}\right)\end{array}$ & $P / l^{\mathrm{b}}\left(\mathrm{CO}_{2}\right)$ \\
\hline PSF & 34 & 32.5 & 0.245 \\
PSF & 2.7 & 30.0 & 3.11 \\
PVC & 20 & 31.8 & 0.014 \\
PVC & 13 & 32.7 & 0.023 \\
PVC & 4 & 12.2 & 0.139 \\
\hline
\end{tabular}

${ }^{a}$ Error within $10 \%$.

${ }^{\mathrm{b}} \mathrm{P} / \mathrm{l}: 10^{-6} \mathrm{~cm}^{3} / \mathrm{cm}^{2} \mathrm{~s} \mathrm{cmHg}$.

selectivity. The surface defects of PSF are much smaller and therefore do not affect the selectivity in gas separation, whereas PAN does not show surface defects at all; due to the fact that PAN is a semicrystalline polymer, an aggregate surface structure has been formed.

The $P / l$ values in Table II show a linear relationship as a function of the reciprocal membrane thickness, except for the $4 \mu \mathrm{m}$-thick PVC film. This is in perfect agreement with the solution-diffusion model: The gas flux is proportional to the reciprocal membrane thickness and selectivity is independent of the membrane thickness.

\section{Pervaporation}

As already described in the Introduction, the pervaporation selectivity seems to become dependent on membrane thickness below a certain thickness. Asymmetric hollow fibers of $\mathrm{PSF}^{5}$ with thin top layers have been prepared and did, by far, not reach the intrinsic selectivity, whereas in the case of gas separation, no reduction in selectivity was observed.

To neglect the influence of the porous support, homogeneous flat membranes have been prepared of PSF, PVC, and PAN. The selectivity and flux as a function of membrane thickness was studied for the dehydration of $80 \mathrm{wt} \%$ acetic acid at $80^{\circ} \mathrm{C}$. The permeate composition as a function of membrane thickness is given in Figure 2. This figure shows that for PSF and PVC as well as for PAN the water concentration in the permeate is constant above a certain minimum thickness. Below this thickness, the water content decreases as the membrane thickness decreases, which results in a drop of the selectivity. It can also be concluded that the effect is large in the case of PSF and PAN and much smaller in the case of PVC. 


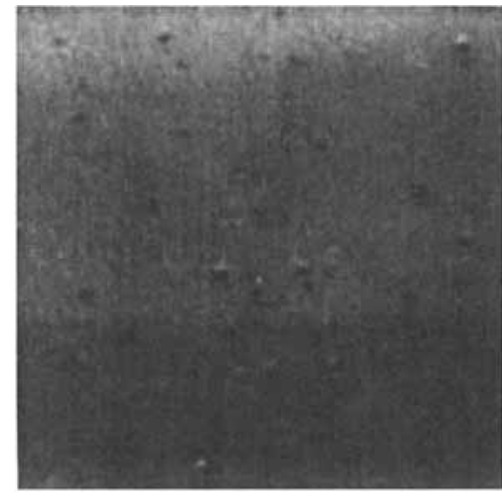

PSF (surface area $3 \times 3 \mu \mathrm{m}$ )

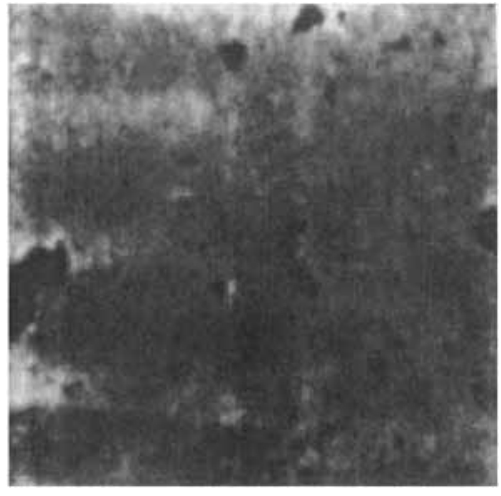

PVC (surface area $6 \times 6 \mu \mathrm{m}$ )

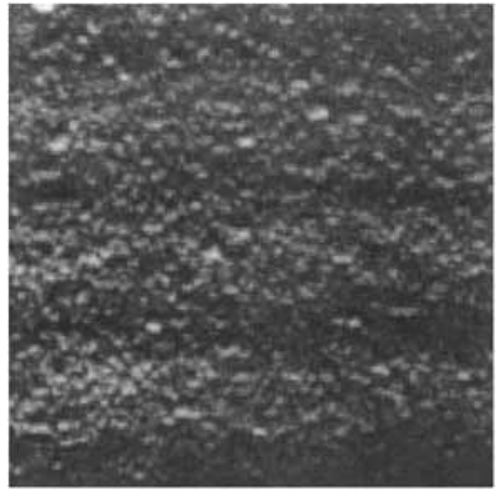

PAN (surface area $6 \times 6 \mu \mathrm{m}$ )

Figure 1 Atomic force microscope pictures of the top-surface of PSF, PVC, and PAN films with a thickness of 6-9 $\mu \mathrm{m}$. PSF and PVC are cast from a tetrahydrofuran solution and PAN from a N,N-dimethylformamide solution.

In Figures 3-5, the total flux as well as the component fluxes are plotted as a function of the reciprocal membrane thickness for PSF, PVC, and PAN, respectively. Many membranes have been prepared and measured, especially for thicknesses below 10 $\mu \mathrm{m}$. Membranes containing pinholes could be easily recognized; the total flux of these membranes was too high and did not fit to the curve of the total flux vs. the reciprocal membrane thickness; besides, the selectivity was extremely low in these cases.

Figures 3(a)-5 (a) show a nice linear relationship between the total flux and the reciprocal membrane thickness as is predicted by the solution-diffusion model (Fickian behavior). By splitting up the total flux in the component fluxes of water and acetic acid, it can be clearly seen how the selectivity de-
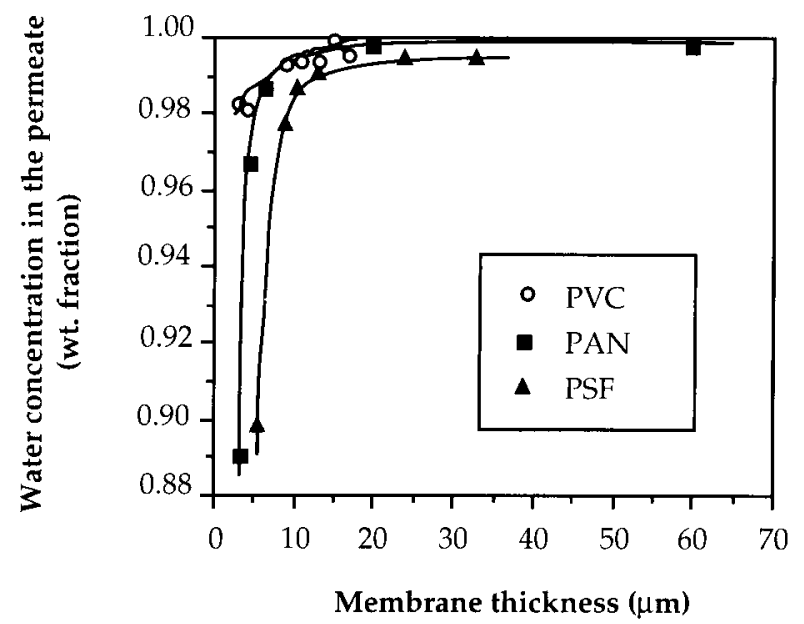

Figure 2 Water content in the permeate as a function of membrane thickness for the dehydration of $80 / 20 \mathrm{wt}$ $\%$ acetic acid/water at $80^{\circ} \mathrm{C}$ by pervaporation. creases with decreasing membrane thickness. The water flux shows a similar Fickian behavior as that of the total flux, whereas the acetic acid flux shows a non-Fickian behavior; the acetic acid flux increases more than proportionally with decreasing membrane thickness in the case of PVC and even exponentially in the cases of PSF and PAN.

Due to the high water selectivity of the membrane material, the acetic acid flux is two orders of magnitude smaller compared to the water flux for a 20 $\mu \mathrm{m}$-thick membrane. Therefore, the total flux is completely controlled by the water flux. Even an exponential increase of the acetic acid flux hardly influences the total flux, but does influence the selectivity.

Pervaporation experiments have also been carried out using pure components as the feed. In Figure 6, the pure water flux and the pure acetic acid flux are given as a function of the reciprocal thickness of a PVC membrane.

Figure 6 shows a Fickian behavior for both components. It can be seen that the pure acetic acid flux is much higher than when a mixture of $80 / 20 \mathrm{wt} \%$ acetic acid/water [ see Fig. 4 (b)] is used as the feed. The addition of water to acetic acid reduces the acetic acid flux by more than two orders of magnitude. This is caused by the lower sorption behavior, but also the cluster formation of acetic acid in the presence of water may contribute to a lower diffusivity.

\section{Membrane Morphology}

In thin-film preparation, the evaporation of the solvent will proceed faster than in the case of thick films. This difference in evaporation rate might re- 


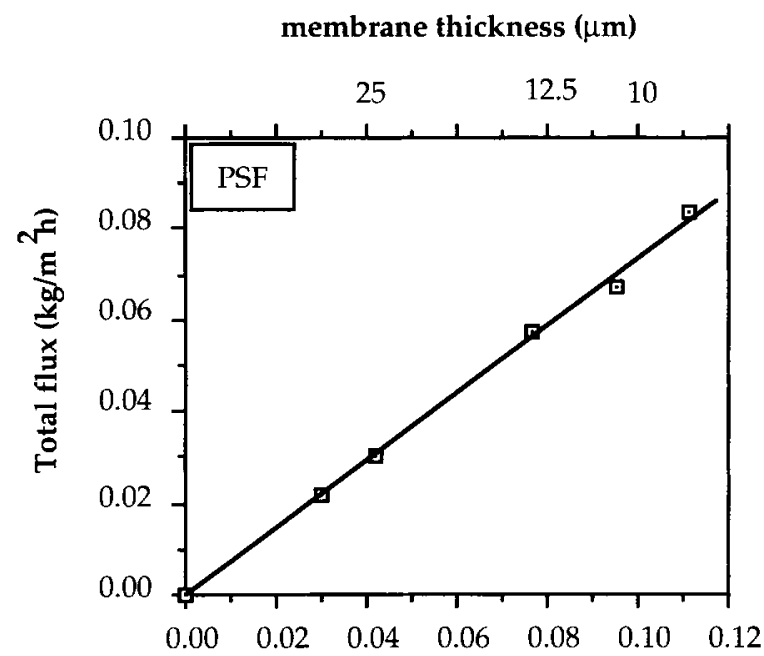

(a) $\quad 1 /$ membrane thickness $(1 / \mu \mathrm{m})$

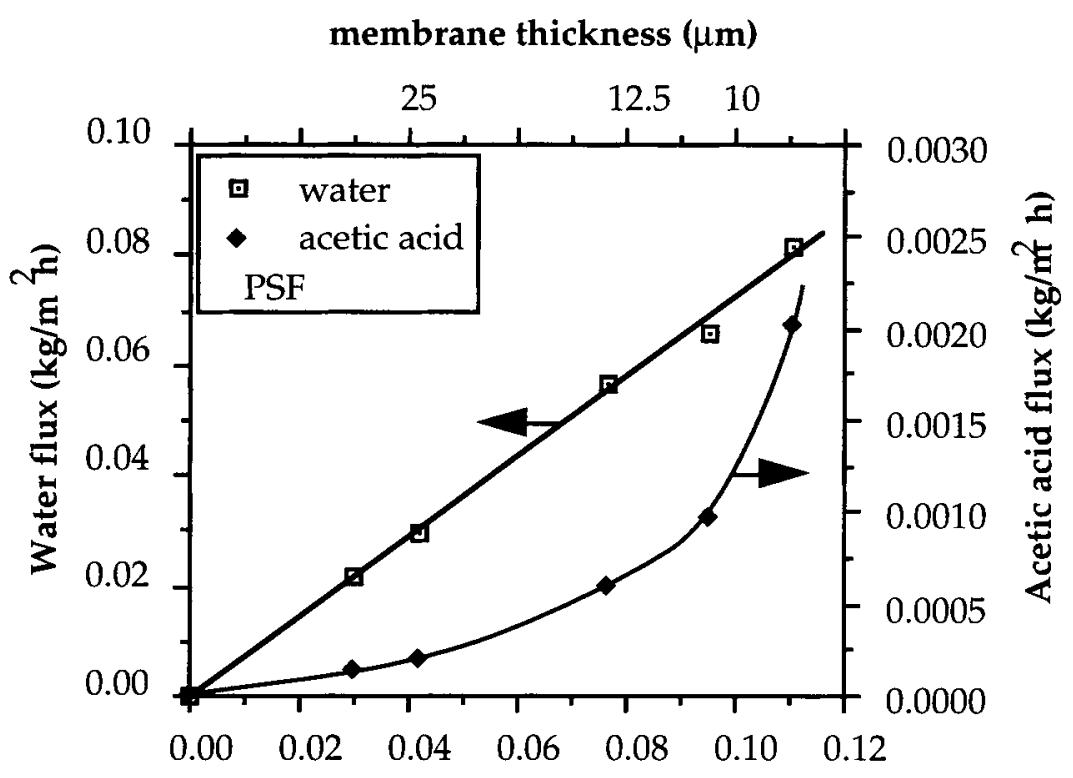

(b) $\quad 1 /$ membrane thickness $(1 / \mu \mathrm{m})$

Figure 3 Total flux (a) and component fluxes (b) as a function of the reciprocal PSF membrane thickness for the dehydration of an $80 / 20 \mathrm{wt} \%$ acetic acid/water mixture at $80^{\circ} \mathrm{C}$; note the different scales on the vertical axis.

sult in a different morphology of the ultimate membrane; a higher rate of evaporation gives the polymer chains less time to achieve the optimal packing. A heat treatment above the glass transition temperature should result in the optimal packing density after all, due to a rearrangement of the polymer chains (relaxation ), and morphology differences will probably disappear. Of the studied polymer membranes, only the PSF membranes were applied to a heat treatment above their glass transition temper- ature $\left(1 \mathrm{~h}\right.$ at $\left.210^{\circ} \mathrm{C}\right)$ in order to improve the resistance against environmental stress-cracking.

To see whether thin films have a different morphology that might explain a lower selectivity, several thin films were stacked together, measured for pervaporation, and compared tc a single membrane of similar total thickness. In Table III, the selectivity and flux are given of single-layer and multilayer membranes of PSF and PVC for the dehydration of $80 \mathrm{wt} \%$ acetic acid at $80^{\circ} \mathrm{C}$. 

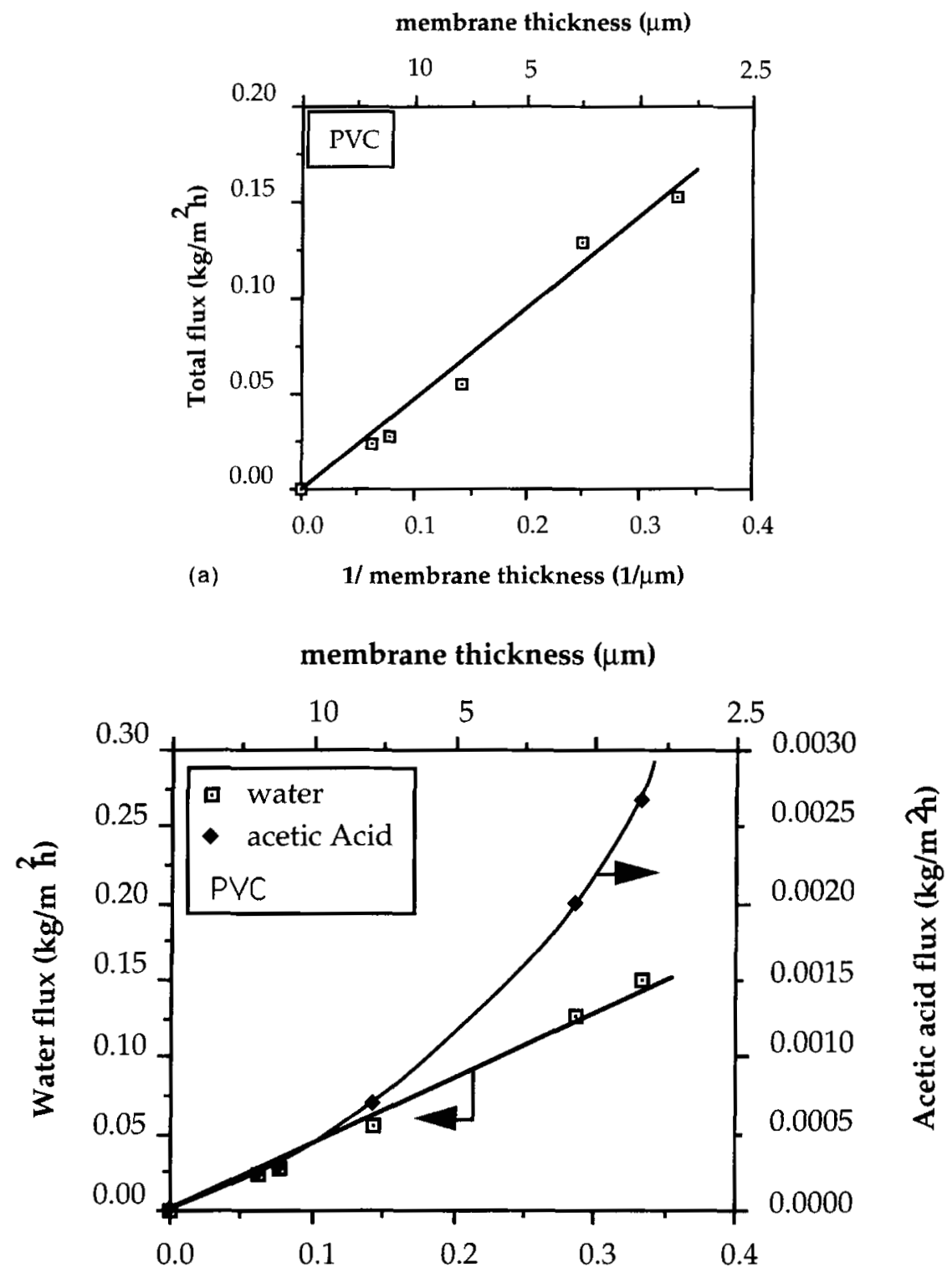

(b)

$1 /$ membrane thickness $(1 / \mu \mathrm{m})$

Figure 4 Total flux (a) and component fluxes (b) as a function of the reciprocal PVC membrane thickness for the dehydration of an $80 / 20 \mathrm{wt} \%$ acetic acid/water mixture at $80^{\circ} \mathrm{C}$.

From Table III it can be seen that in the case of PVC the selectivity of the multilayer membranes are similar to that of a (single-layer) membrane with an equal total thickness. However, the selectivity of one membrane with the same thickness as one layer of the multilayer membrane is lower.

The permeate composition of the PSF multilayer membrane is in between the value of the thin and the thick single-layer membrane. If the morphology determines the low selectivity of thin membranes, the selectivity of the multilayer membrane should have the same value as that of the thin membrane. Therefore, a lower selectivity for thin membranes cannot be explained by a difference in polymer morphology. Besides, morphology differences should not be present anymore in the PSF membranes due to the heat treatment above the glass transition temperature; nevertheless, the selectivity of these membranes appeared to be dependent on thickness as well (see Fig. 2). 

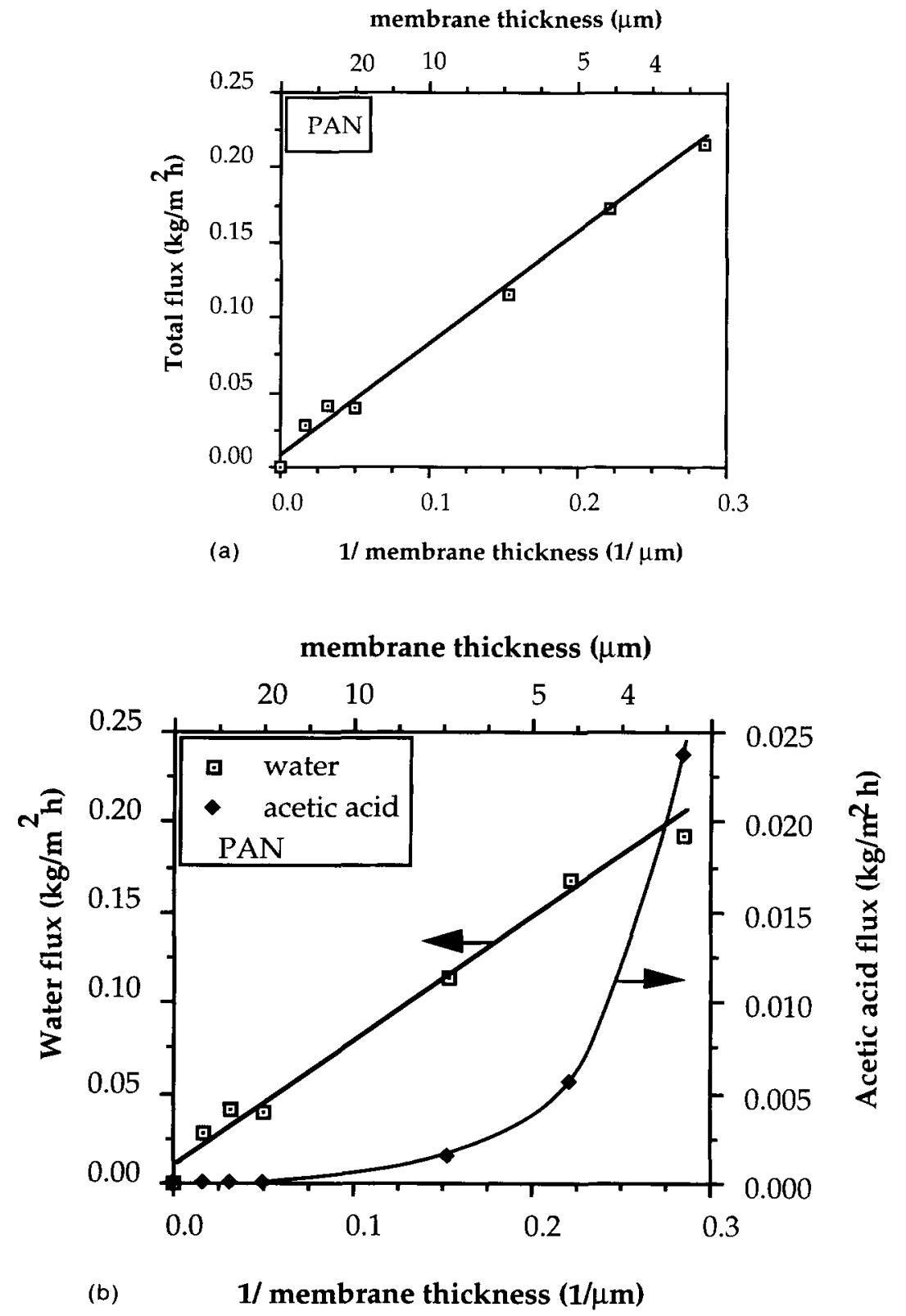

Figure 5 Total flux (a) and component fluxes (b) as a function of the reciprocal PAN membrane thickness for the dehydration of an $80 / 20 \mathrm{wt} \%$ acetic acid/water mixture at $80^{\circ} \mathrm{C}$.

These multilayer experiments together with the pervaporation experiments, where the selectivity of homogeneous PSF, PVC, and PAN was studied as a function of membrane thickness, confirm the hypothesis that selectivity becomes dependent on the membrane thickness below a certain limiting thickness and is not determined by morphology differences. This limiting thickness has a value of around $20 \mu \mathrm{m}$. Above this thickness, selectivity is independent of the membrane thickness, which is in accordance with the solution-diffusion theory.
The defects observed in PVC films by atomic force microscopy that result in a lowering of the selectivity for gas separation when thin films are used might also be responsible for the decrease in selectivity observed for pervaporation using thin films. With PSF membranes, the selectivity is found to be independent of membrane thickness for gas separation, whereas in the case of pervaporation, a thickness dependency has been observed. An explanation for this phenomenon has to be found in differences in sorption and diffusion between pervaporation and 


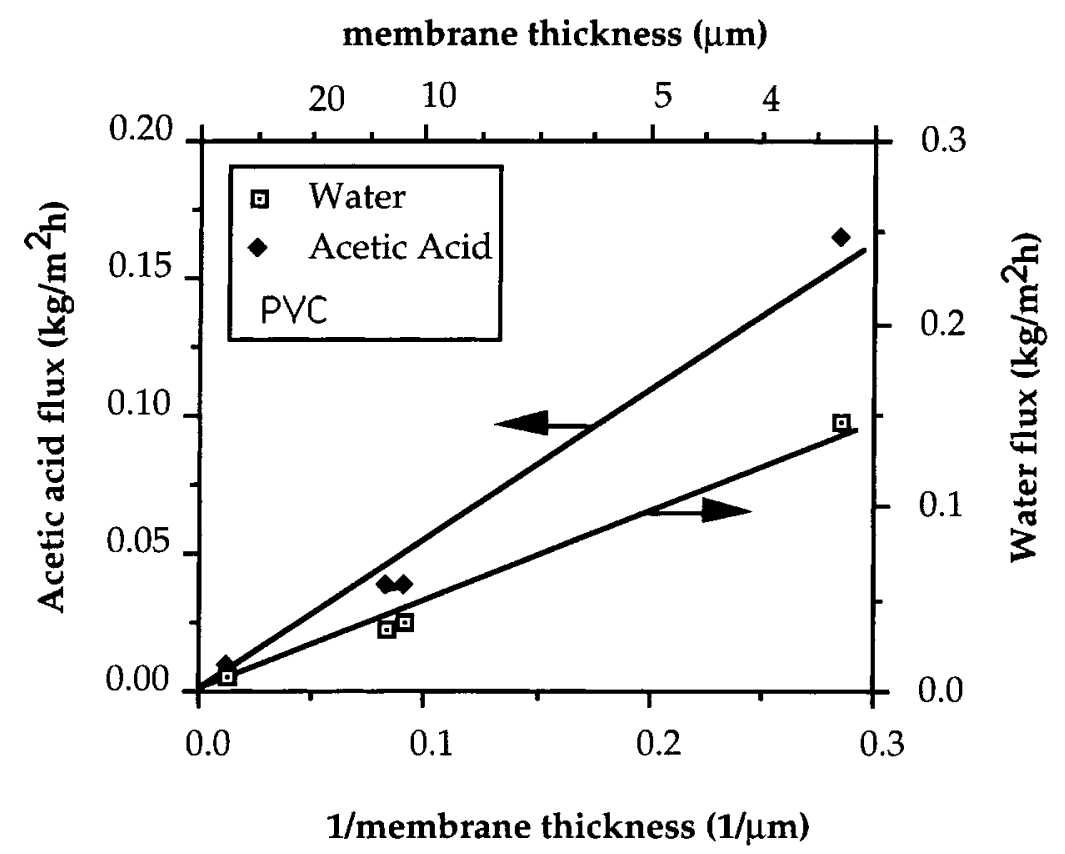

Figure 6 Pure water flux and pure acetic acid flux as a function of the reciprocal membrane thickness for PVC.

gas separation. The influence of desorption is neglected.

Comparing pervaporation and gas separation, three major differences can be distinguished, viz., relatively strong mutual interactions between the various components, coupling of the permeating components, and a higher affinity (thermodynamic interaction) between penetrants and polymer, all occurring in the case of pervaporation.

\section{Thermodynamic Interaction and Coupling}

Phenomena that can be neglected in gas separation but might play an important role in pervaporation are thermodynamic interaction and coupling. Ther- modynamic interaction represents the interaction of the permeating components with the membrane material and coupling represents the influence of the flow of one component on the flow of the other components.

Thermodynamic interaction plays an important role in sorption selectivity. A higher affinity of the membrane material with component $i$ compared to component $j$ mostly results in preferential sorption of component $i$; besides interaction, free entropy of mixing plays a role as well. In Table IV, the sorption selectivities of PSF, PAN, and PVC for two different acetic acid/water mixtures are represented.

From Table IV, it can be concluded that PSF and PVC absorb acetic acid preferentially, despite the

Table III Selectivity and Flux of PSF and PVC Single- and Multilayer Membranes of Different Thicknesses for the Dehydration of $80 \mathrm{Wt} \%$ Acetic Acid at $80^{\circ} \mathrm{C}$

\begin{tabular}{lccccccc}
\hline & $\begin{array}{c}\text { Layer } \\
\text { Thickness } \\
(\mu \mathrm{m})\end{array}$ & $\begin{array}{c}\text { No. of } \\
\text { Layers }\end{array}$ & $\begin{array}{c}\text { Total } \\
\text { Thickness } \\
(\mu \mathrm{m})\end{array}$ & $\begin{array}{c}\text { Feed } \\
(\text { Wt \% Acid) }\end{array}$ & $\begin{array}{c}\text { Permeate } \\
(\text { Wt \% Acid) }\end{array}$ & $\begin{array}{c}\text { Flux } \\
\left(\mathrm{kg} / \mathrm{m}^{2} \mathrm{~h}\right)\end{array}$ \\
\hline PSF & 33 & 1 & 33 & 80.30 & 0.60 & 675 \\
PSF & 9 & 4 & 36 & 79.60 & 1.50 & 256 & 0.022 \\
PSF & 9 & 1 & 9 & 80.10 & 2.40 & 164 & 0.084 \\
PVC & 11 & 1 & 11 & 79.50 & 0.80 & 481 \\
PVC & 3 & 4 & 12 & 79.70 & 1.00 & 389 & 0.034 \\
PVC & 3 & 1 & 3 & 80.40 & 1.70 & 237 & 0.152 \\
\hline
\end{tabular}


Table IV Sorption Selectivity of PSF, PAN, and $P V C$ as a Function of the Acetic Acid/Water Composition

\begin{tabular}{lcc}
\hline Polymer & $\begin{array}{c}\text { Water in Feed } \\
(\mathrm{Wt} \%)\end{array}$ & $\begin{array}{c}\text { Sorption Selectivity } \\
\left(\alpha_{\mathrm{H}_{2} \mathrm{O}}^{S}\right)\end{array}$ \\
\hline PSF & 10 & 0.58 \\
PSF & 20 & 0.44 \\
PAN & 10 & 1.47 \\
PAN & 20 & 1.06 \\
PVC & 10 & 0.86 \\
PVC & 20 & 0.71 \\
\hline
\end{tabular}

fact that they are highly water selective in pervaporation. PAN absorbs water preferentially, but has only a low sorption selectivity. This means that pervaporation selectivity is completely determined by the difference in diffusion of water and acetic acid; water, the smaller component, has a higher diffusion rate than that of acetic acid.

The affinity between the components and the polymer is rather low as can be concluded from the relatively low degree of sorption $\left(\leq 8 \mathrm{wt} \%{ }^{10}\right)$. On the other hand, the interaction between acetic acid and water may be quite high due to the hydrogenbonding ability between the two components. Therefore, a strong influence of the faster permeating water molecules on the relatively slowly permeating acetic acid molecules may be expected. The higher the water flux (thin membranes), the more acetic acid will be dragged along by the water flux, and because the water flux is much higher than is the acetic acid flux, the effect of the latter on the water flux will be negligible. By making the membrane thinner, the flux of both components will increase, but due to coupling, an extra flux increase of the slowest permeating component can be expected. To explain a decrease in selectivity as a function of membrane thickness due to flow coupling, transport relations are derived in terms of a friction model.

The transport equation for component $i$ of a binary mixture permeating through a membrane can be expressed in terms of driving forces and friction: ${ }^{12}$

$-($ driving force on $i)=($ friction of $j$ on $i)$

$+($ friction of membrane on $i)$

The following Maxwell-Stefan equations can be derived for a binary mixture of components $i$ and $j$ permeating through a polymer:

$$
\begin{aligned}
& \frac{1}{R T} \frac{d\left(\mu_{i}\right)}{d z}=\frac{\left(v_{j}-v_{i}\right)}{D_{i j}}-\frac{v_{i}}{D_{i m}} \\
& \frac{1}{R T} \frac{d\left(\mu_{j}\right)}{d z}=\frac{\left(v_{i}-v_{j}\right)}{D_{j i}}-\frac{v_{j}}{D_{j m}}
\end{aligned}
$$

Substitution of the chemical potentials by the activities results in the relations

$$
\begin{aligned}
& \frac{d\left(\ln a_{i}\right)}{d z}=\frac{x_{j}\left(v_{j}-v_{i}\right)}{D_{i j}}-\frac{\left(x_{m} v_{i}\right)}{D_{i m}} \\
& \frac{d\left(\ln a_{j}\right)}{d z}=\frac{x_{i}\left(v_{i}-v_{j}\right)}{D_{j i}}-\frac{\left(x_{m} v_{j}\right)}{D_{j m}}
\end{aligned}
$$

with $J_{i}=v_{i} \bar{C}_{i}, J_{j}=v_{j} \bar{C}_{j},\left(x_{m} / D_{i m}\right)=\left(1 / D_{i m}^{\prime}\right)$ and $\left(x_{m} / D_{j m}\right)=\left(1 / D_{j m}^{\prime}\right)$. The component fluxes can be expressed as

$$
\begin{aligned}
& J_{i}=\left[\frac{\left(\bar{C}_{i} D_{i j} D_{i m}^{\prime}\right)}{a_{i}\left(x_{j} D_{i m}^{\prime}+D_{i j}\right)}\right]\left[\frac{\left(J_{j} a_{i} x_{j}\right)}{\left(\bar{C}_{j} D_{i j}\right)}-\frac{d a_{i}}{d z}\right] \\
& J_{j}=\left[\frac{\left(\bar{C}_{j} D_{j i} D_{j m}^{\prime}\right)}{a_{j}\left(x_{i} D_{j m}^{\prime}+D_{j i}\right)}\right]\left[\frac{\left(J_{i} a_{j} x_{i}\right)}{\left(\bar{C}_{i} D_{j i}\right)}-\frac{d a_{j}}{d z}\right]
\end{aligned}
$$

These relations are similar to the relations derived by Kedem $^{13}$ :

$$
\bar{P}_{i}=\left[\frac{\left(\bar{C}_{i} D_{i j} D_{i m}^{\prime}\right)}{\left(x_{j} D_{i m}^{\prime}+D_{i j}\right)}\right] ; \quad \bar{P}_{j}=\left[\frac{\left(\bar{C}_{j} D_{j i} D_{j m}^{\prime}\right)}{\left(x_{i} D_{j m}^{\prime}+D_{j i}\right)}\right]
$$

$\bar{P}_{i}$ and $\bar{P}_{j}$ represent the permeability coefficient of component $i$ and $j$, respectively.

$$
\bar{Q}=\frac{x_{j}}{\left(\bar{C}_{j} D_{i j}\right)}=\frac{x_{i}}{\left(\bar{C}_{i} D_{j i}\right)}
$$

represents the mutual drag coefficient.

Relations ( $3 a$ ) and ( $3 b$ ) can now be expressed as

$$
\begin{aligned}
& J_{i}=-\frac{\bar{P}_{i}}{a_{i}} \frac{d\left(a_{i}\right)}{d z}+\bar{Q} \bar{P}_{i} J_{j} \\
& J_{j}=-\frac{\bar{P}_{j}}{a_{j}} \frac{d\left(a_{j}\right)}{d z}+\bar{Q} \bar{P}_{j} J_{i}
\end{aligned}
$$

There is no direct relation between $\bar{P}$ and the membrane thickness or between $\bar{Q}$ and the membrane thickness.

Assuming constant coefficient integration of relations (4a) and ( $4 b)$ gives 


$$
\begin{gathered}
J_{i}=\frac{\bar{P}_{i}}{\Delta z} \ln \left(\frac{a_{i}^{\prime}}{a_{i}^{\prime \prime}}\right)+\bar{Q} \bar{P}_{i} J_{j} \\
J_{j}=\frac{\bar{P}_{j}}{\Delta z} \ln \left(\frac{a_{j}^{\prime}}{a_{j}^{\prime \prime}}\right)+\bar{Q} \bar{P}_{j} J_{i}
\end{gathered}
$$

In the case of highly selective membranes, $J_{i}$ $\gg J_{j}$ and, therefore, the last term on the right-hand side of relation ( $5 \mathrm{a}$ ) can be neglected. By changing the membrane thickness $\bar{P}, \bar{Q}$ and the driving force remain constant. To study the influence of flow coupling on the component fluxes as a function of the membrane thickness, relations (5a) and (5b) can be simplified by

$$
\begin{aligned}
& J_{i}=\frac{C_{1}}{\Delta z} \\
& J_{j}=\frac{C_{2}}{\Delta z}+C_{3} J_{i}=\frac{C_{2}}{\Delta z}+C_{3} \frac{C_{1}}{\Delta z}
\end{aligned}
$$

From relations (6a) and ( $6 b$ ), it can be concluded that the flux of the faster permeating component $i$ influences the flux of $j$ when $C_{3}$ is relatively large, which means a large mutual interaction between components $i$ and $j$.

Furthermore, it can be concluded that both component fluxes are proportionally related to the reciprocal membrane thickness and that the ratio of the component fluxes is constant and independent of the membrane thickness. Therefore, flow coupling cannot explain the decrease in selectivity using thin membranes, unless $\bar{Q}$ and/or $\bar{P}$ are dependent on the membrane thickness.

That this is not unrealistic might be concluded from the observations by Park, ${ }^{14}$ Mandelkern and Long, ${ }^{15}$ and Ware and Cohen. ${ }^{16}$ They observed independently that at nonequilibrium conditions thin films absorb more penetrant molecules per surface area than do thick films. Ware and Cohen also found that this occurs below a limiting thickness. This indicates that during pervaporation at steady state the degree of swelling of thin membranes might be larger than of thick films. If this is true, the average concentration $\bar{C}_{i}$ will be higher for thin films below a limiting thickness, which results in variable values for $\bar{Q}$ and $\bar{P}$ as a function of the membrane thickness.

\section{Sorption Resistance}

Kim and Kammermeyer ${ }^{17}$ observed a sorption resistance at the liquid-polymer interface for permeation of a single component through thin films. This resistance increases with decreasing membrane thickness and results in a lower surface concentration of the permeant compared to the free equilibrium sorption concentration. Due to a reduced driving force, a lower flux is obtained.

In the case of acetic acid/water mixtures, only a sorption resistance for water might explain a decrease in selectivity. However, water shows a perfect Fickian behavior with decreasing membrane thickness, whereas the acetic acid flux increases more than proportionally. Therefore, a sorption resistance cannot explain the decrease in selectivity in the case of acetic acid/water separation using thin films.

\section{Sorption-induced Defects}

The additional increase of the acetic acid flux with decreasing membrane thickness might, of course, be the result of small defects in the membrane. However, directly after membrane preparation, only in the case of thin $( \pm 4 \mu \mathrm{m})$ PVC membranes, defects were present large enough to affect the selectivity. This can be concluded from gas separation experiments and atomic force microscopy results. In the case of PSF and PAN, we conclude that defects must then be introduced during pervaporation.

Crazes and cracks can be formed in polymeric materials once a critical strain $\epsilon_{i}$ has been reached. The formation of crazes is enhanced in a chemically active environment due to plasticization of the material and the reduction of the surface energy of the craze fibrils and, in the end, might even result in material failure (cracks). The crazing agent acts through its presence within the polymer matrix. By increasing the chain mobility (lowering $T_{g}$ ), the penetrant facilitates the primary and secondary steps of craze initiation: nucleation and stabilization of a craze. It causes the lowering of critical strain and stress in polymers such as polystyrene, PSF,

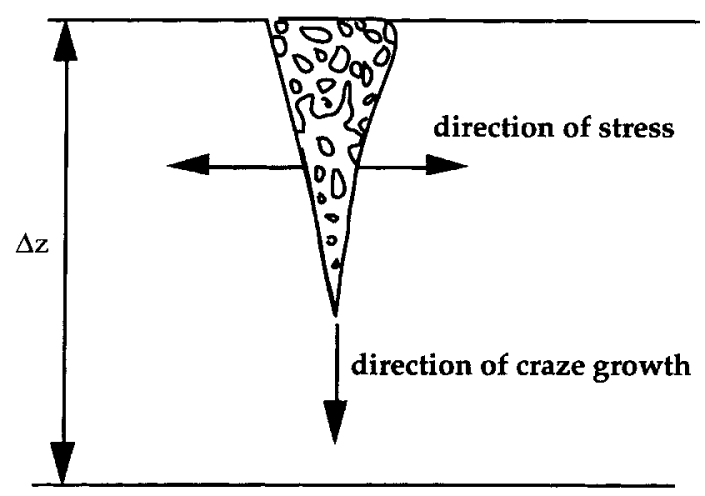

Figure 7 Schematic representation of a craze in a polymer film. 
PVC, poly (phenylene oxide), or polycarbonate. ${ }^{18}$ PSF, e.g., shows a reduction of critical strain by more than a factor 10 , from $2.5 \%$ in air to $\pm 0.2 \%$ in organic liquids with a solubility parameter between 9 and 12. ${ }^{19}$ Therefore, craze initiation can be defined as the localized collective dilatational reorganization of stiff chain segments accompanied by matrix breakup. ${ }^{18}$

Material failure has been observed for relatively thick PSF and poly (ether sulfone) membranes ${ }^{11}$ when failure occurred at the place where the O-ring is pressing on the membrane. Hopfenberg ${ }^{20}$ observed solvent-induced crazes at the swelling front when he examined cross sections of polystyrene samples, which had been immersed in $n$-alkane. During the beginning of the sorption process, large concentration differences can be observed across the membrane, resulting in stress formation transverse to the direction of diffusion ${ }^{21}$ (see Fig. 7). The swollen region generates tensile forces on the unswollen, glassy region, whereas the glassy region causes compressive forces on the swollen region.

Comyn ${ }^{22}$ described examples of the formation of microcavities due to environmental stress-cracking and Drioli et al. ${ }^{23}$ described permeability experiments carried out with polystyrene, polycarbonate, and acrylonitrile-butadiene-styrene membranes with and without the presence of crazes (generated by applying a high pressure on the polymer film). They observed permeability through these crazes as well as a reduction of crazes by the presence of glass beads in the polymer and upon approaching the glass transition temperature of the polymer. The disappearance of crazes at the glass transition tempera-

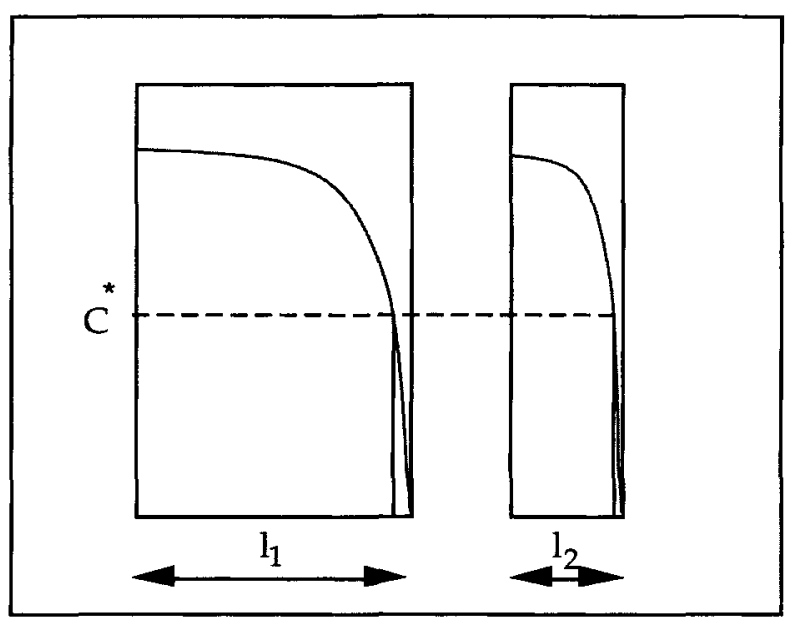

Figure 8 Schematic representation of the same concentration profile of a single component in two membranes of different thickness. ture has also been observed by Nicolais and $\mathrm{Di}$ Benedetto $^{24}$ and by Salee. ${ }^{25}$

In the case of pervaporation, large concentration differences across the membrane are present, which might result in the presence of stresses analogous to the nonequilibrium stage of sorption. Due to these stresses and the plasticizing effect of the acetic acid/ water solution, crazes might be initiated and grow in the direction of diffusion as long as there is enough liquid present at the tip of the craze.

Due to the low pressure at the permeate side and the relatively unswollen state of the membrane, the craze will stop growing at that distance in the membrane where the concentration of the permeating liquid becomes too low (decreased plasticization). In other words, the layer at the permeate side that contains a liquid concentration lower than a critical concentration $C^{*}$ is responsible for the blocking of craze growth. In case the mechanical strength of this layer is too small, crazes may break through and could result in defects of the membrane that cover the whole membrane thickness.

In Figure 8, a concentration profile is given for a thick and for a thin membrane. According to the solution-diffusion theory, the concentration as a function of the relative distance in the membrane should be the same for both membranes. From this figure, it can be concluded that the layer with a liquid concentration lower than $C^{*}$ and responsible for blocking of craze growth is thinner in the case of a thin membrane compared to that in a thick membrane. To explain (below a certain membrane thickness) the strong flux increase of acetic acid with decreasing membrane thickness, one might conclude that a limiting thickness of the relatively unswollen layer is required to stop the craze from growing. Below this thickness, crazes might break through due to a lack of mechanical stability. The thinner the membrane, the more crazes will break through and the higher the contribution of craze flux to the total membrane flux will be.

The craze flux is very small compared to the total membrane flux and will increase both component fluxes. Because the acetic acid flux for pervaporation is so small in comparison with the water flux, the addition of a small but almost equal craze flux for both components to the pervaporation flux will have a larger influence on the acetic acid flux than on the water flux. The size of these crazes can be as large as $200-3000 \AA$, which has been observed for polystyrene. $^{23}$

From the results presented in Figures 2-5, it was concluded that the effect of selectivity decrease for PVC is much less than for PSF and PAN. Since the 
pervaporation experiments have been carried out at $80^{\circ} \mathrm{C}$ and the glass transition temperatures of PVC, PAN, and PSF according to literature ${ }^{26}$ are 87,120 , and $190^{\circ} \mathrm{C}$, respectively, this is in good agreement with the observations of craze reduction upon approaching the glass transition temperature.

Let us assume that crazes are formed and that they result in defects (cracks) that can be compared with capillary pores. In the case of pervaporation, the flux through capillaries can be described by the Knudsen flow. The flux for component $i$ can then be expressed by

$$
J_{i, \text { total }}(z)=J_{i, \text { pervap. }}(z)+J_{i, \text { Knudsen }}(z)
$$

with

$$
\begin{gathered}
J_{i, \mathrm{Knudsen}}(z)=0 \text { for } z \geq 20 \mu \mathrm{m} \\
J_{i, \mathrm{Knudsen}}(z)=\frac{1}{3} d_{p} \cdot \sqrt{\frac{8}{\pi R T M_{i}}} \cdot n \cdot\left(\frac{A_{p}}{A_{m}}\right) \cdot \frac{d\left(p_{i}\right)}{d z} \\
\text { for } z<20 \mu \mathrm{m}
\end{gathered}
$$

The flux contribution due to pervaporation can be calculated from the flux obtained for a membrane with a thickness of $20 \mu \mathrm{m}$ using the relation

$$
\frac{J_{i, \text { pervap. }}(z)}{J_{i, \text { total }}(20 \mu \mathrm{m})}=\frac{1 / z}{1 / 20}=\frac{20}{z}
$$

For membranes thinner than $20 \mu \mathrm{m}$, the total acetic acid flux and water flux were measured (see
Figs. 3-5) and the pervaporation fluxes were calculated with the help of eq. (10). The flux contribution due to the Knudsen flow can now be calculated from the experimentally obtained flux by choosing a fixed value for the pore diameter $d_{\text {pore }}$ and taking the number of pores $n$ as a fitting parameter. This was done for the acetic acid flux with $d_{\text {pore }}=50 \mathrm{~nm}$ (this pore size is a chosen value) With the number of pores found by fitting the acetic acid flux and a pore diameter of $50 \mathrm{~nm}$, the total water flux can also be calculated and compared with the total water flux that was measured. To meet the measured acetic acid flux, the number of pores found for the thinnest membranes should be 176.688 per $\mathrm{cm}^{2}$ for a $3.5 \mu \mathrm{m}$ PAN membrane, 28.728 per $\mathrm{cm}^{2}$ for a $9 \mu \mathrm{m}$ PSF membrane, and 12.528 per $\mathrm{cm}^{2}$ for a $3 \mu \mathrm{m}$ PVC membrane. If the pore size is fixed at a smaller value, the number of pores increases. These calculations only show whether the pervaporation flux of water as a function of the reciprocal membrane thickness will be influenced by the extra contribution of transport through the number of capillaries calculated from the fitting procedure.

The measured water fluxes (squares) and the calculated water fluxes from the model (dashed lines) are plotted together in Figure 9. From this figure, it can be concluded that the calculated water fluxes show a linear relationship with the reciprocal membrane thickness and that they seem to be in good agreement with the experimentally obtained values. The extra flux through the capillaries is so small that it does not influence the pervaporation flux of water.

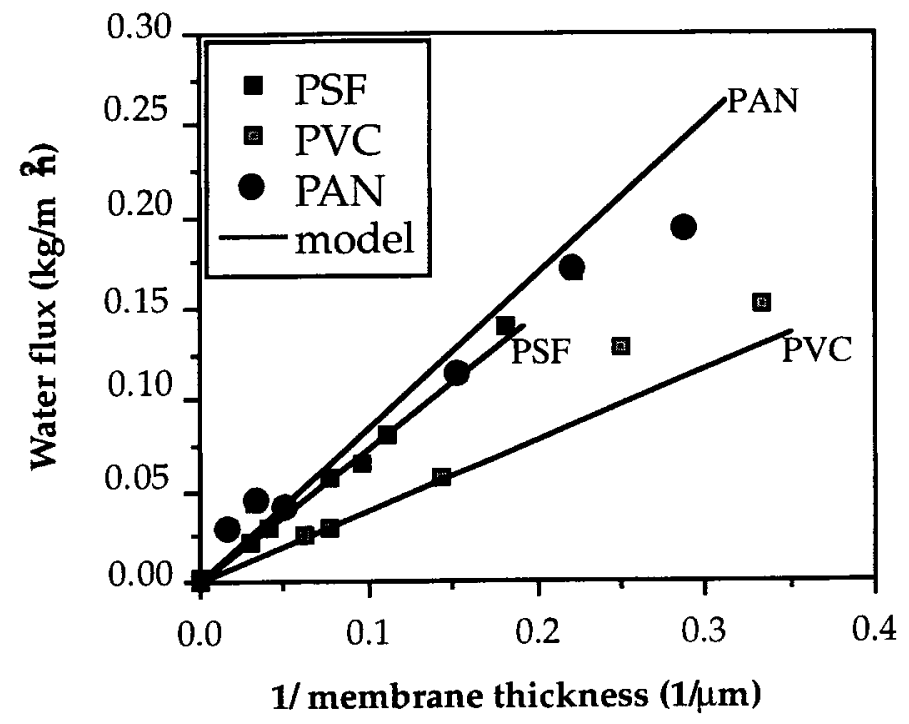

Figure 9 Experimentally obtained water fluxes (squares) and calculated water fluxes (dashed lines) as a function of the reciprocal membrane thickness for PSF, PVC, and PAN. 
A calculated decrease in selectivity with decreasing membrane thickness could also be obtained from the water/acetic acid ratio in the permeates calculated by the model. Only for membranes thinner than 5-6 $\mu \mathrm{m}$ do the calculated values start to deviate a little, although not systematically, from the experimental values. At these thicknesses, the contribution of the Knudsen flow is relatively large, from which it might also be concluded that a different kind of flow other than the Knudsen flow takes place through the crazes (e.g., surface diffusion).

To gain more evidence for the existence of solvent-induced defects in thin membranes, pervaporation experiments have to be carried out at variable feed pressures. The pervaporation flux will hardly be influenced, but the flux due to the Knudsen flow will.

\section{CONCLUSIONS}

From gas separation and pervaporation experiments with PSF, PVC, and PAN membranes of variable thicknesses, it can be concluded that gas separation selectivity is independent of the membrane thickness as is described by the solution-diffusion theory, and that in the case of pervaporation, the selectivity decreases with decreasing membrane thickness below a limiting thickness $(\sim 15 \mu \mathrm{m})$.

The decrease of selectivity as a function of the membrane thickness could not be explained by differences in polymer morphology, nor by sorption resistances.

This dependence of selectivity on the membrane thickness was analyzed with respect to flow coupling, but, again, could not be explained from this effect. Only if the mutual drag coefficient $Q$ or the permeability coefficient $P$ increase with decreasing membrane thickness can a selectivity decrease be explained.

The formation of defects during pervaporation due to solvent-induced craze formation was discussed. Transport through these defects could be well described by the Knudsen flow. By fitting the number of pores as a function of the membrane thickness, the calculated water fluxes are in good agreement with the experimentally obtained values. Only at very small thicknesses do the calculated values deviate from the experimental ones.

Although the phenomenon of decreasing selectivity in pervaporation upon decreasing membrane thickness is not yet completely understood, its appearance has been clearly proven.

The work described in this paper is part of the Briteproject RI1B-196. The European Community (BRITE), B.P. Chemicals Ltd., and Deutsche Carbone GmbH Geschäftseinheit GFT are gratefully acknowledged for their financial support.

\section{LIST OF SYMBOLS}

\section{Symbol}

$\begin{array}{lll}a & \begin{array}{l}\text { activity } \\ \text { activity feed, activity } \\ a^{\prime}, a^{\prime \prime}\end{array} \\ & \begin{array}{l}\text { permeate } \\ \text { surface area }\end{array} & \mathrm{m}^{2} \\ C & \begin{array}{l}\text { concentration } \\ \text { diameter }\end{array} & \mathrm{mol} / \mathrm{m}^{3} \\ d & \text { diffusivity } \\ D & \text { flux } & \mathrm{m} / \mathrm{s} \\ J & \text { molecular weight } & \mathrm{mol} / \mathrm{s} \\ M & \text { number of pores } & \\ n & \text { pressure } & \mathrm{N} / \mathrm{m}^{2} \\ p & \text { mutual drag coefficient } \\ Q & \mathrm{~m} \mathrm{~s} / \mathrm{mol} \\ R & \text { gas constant } & \mathrm{J} / \mathrm{mol} \mathrm{K} \\ P & \text { permeability coefficient } & \mathrm{mol} / \mathrm{m} \mathrm{s} \\ T & \text { temperature } & \mathrm{K} \\ v & \text { velocity } & \mathrm{m} / \mathrm{s} \\ x & \text { mol fraction } & \\ z & \text { thickness, distance } & \mathrm{m} \\ \mu & \text { chemical potential } & \mathrm{J} / \mathrm{mol}\end{array}$

Indices

$\begin{array}{llll}\text { av } & \text { average } & \mathbf{m} & \text { membrane } \\ i, j & \text { components } i \text { and } j & \mathbf{p} & \text { pore }\end{array}$

\section{REFERENCES}

1. H. K. Lonsdale, U. Merten, and R. L. Riley, J. Appl. Polym. Sci., 9, 1341 (1965).

2. R. C. Binning, R. J. Lee, J. F. Jennings, and E. C. Martin, Ind. Eng. Chem., 53 (1), 45-50 (1961).

3. J. P. Brun, G. Bulvestre, A. Kergreis, and M. Guillou, J. Appl. Polym. Sci., 18, 1663-1683 (1974).

4. J. W. F. Spitzen, G. H. Koops, M. H. V. Mulder, and C. A. Smolders, in Proceedings of the 3rd International Conference on Pervaporation Processes in the Chemical Industry, R. Bakish, Ed., Bakish Materials Corporation, Englewood, New Jersey. 1988, pp. 252-257.

5. G. H. Koops, M. H. V. Mulder, and C. A. Smolders, to appear.

6. P. Aptel, J. Cuny, J. Jozefonvicz, G. Morel, and J. Neel, J. Appl. Polym. Sci., 18, 351-364 (1974).

7. M. H. V. Mulder, PhD Thesis, University of Twente, The Netherlands, 1984.

8. I. Pinnau and W. J. Koros, U.S. Pat. 4,902,422 (1990). 
9. M. H. V. Mulder, C. Valentini, and R. Siste, Lecture presented at the PMST Symposium, Enschede, The Netherlands, June 1991, to appear.

10. G. H. Koops, PhD Thesis, University of Twente, Enschede, The Netherlands, 1992, Chap. 2.

11. M. H. V. Mulder, F. Kruitz, and C. A. Smolders, J. Memb. Sci., 11, 349 (1982).

12. J. A. Wesselingh and R. Krishna, Mass Transfer, Ellis Horwood, London, 1990.

13. O. Kedem, J. Membr. Sci., 47, 277-284 (1989).

14. G. S. Park, J. Polym. Sci., 11 (2), 97-115 (1953).

15. L. Mandelkern and F. Long, J. Polym. Sci., 6, 457 (1951).

16. R. A. Ware and C. Cohen, J. Appl. Polym. Sci., 25, 717-729 (1980).

17. S. M. Kim and K. Kammermeyer, Sep. Sci., 5(6), 679-697 (1970).

18. H. H. Kausch, Polymer Fracture; Polymers/Properties and Applications 2, Springer-Verlag, Berlin, 1987.
19. R. P. Kambour, E. E. Romagosa, and C. L. Gruner, Macromolecules, 5, 335-340 (1972).

20. H. B. Hopfenberg, J. Membr. Sci., 3, 215-230 (1978).

21. J. H. Petropoulos and P. P. Roussis, J. Membr. Sci., 3, 343-356 (1978).

22. J. Comyn, Progr. Rubber Plast. Techn., 1(3), 1-13 (1985).

23. E. Drioli, L. Nicolais, and A. Ciferri, J. Polym. Sci., 11, 3327-3329 (1973).

24. L. Nicolais and A. T. Di Benedetto, J. Appl. Polym. Sci., 15, 1585 (1971).

25. G. Salee, J. Appl. Polym. Sci., 15, 2049 (1971).

26. A. E. Schouten and A. K. van der Vegt, Plastics, Delta Press, Amerongen, The Netherlands 1987, p. 149.

Received December 11, 1992

Accepted March 22, 1994 